\title{
'Thing' and 'non-thing' ontologies
}

\author{
Michael Esfeld \\ University of Lausanne, Department of Philosophy \\ Michael-Andreas.Esfeld@unil.ch,www.michaelesfeld.com \\ (draft 21 December 2017)
}

(for Ricki Bliss and James Miller (eds.): Routledge handbook of metametaphysics)

\section{Discrete objects: from Aristotelian metaphysics to ontic structural realism}

Thing ontologies are ontologies that consider the universe to be made up of a plurality of discrete objects. Non-thing ontologies can take the form of ontologies of discrete objects, too, but not necessarily so: they can also be conceived as ontologies of one continuous object. Let us consider the central versions of ontologies of discrete objects first, starting with thing ontologies, moving from there to non-thing ontologies of discrete objects and finally ontologies of one continuous object.

The paradigm example of a thing ontology is atomism, which can be traced back to the Presocratics. Thus, Democritos is reported as maintaining

That substances infinite in number and indestructible, and moreover without action or affection,

travel scattered about in the void. When they encounter each other, collide, or become entangled, collections of them appear as water or fire, plant or man. (Fragment Diels-Kranz 68 A57, translation taken from Graham 2010, p. 537)

At our time, Feynman says in the introduction to the Feynman lectures on physics:

If, in some cataclysm, all of scientific knowledge were to be destroyed, and only one sentence passed on to the next generations of creatures, what statement would contain the most information in the fewest words? I believe it is the atomic hypothesis (or the atomic fact, or whatever you wish to call it) that all things are made of atoms - little particles that move around in perpetual motion, attracting each other when they are a little distance apart, but repelling upon being squeezed into one another. In that one sentence, you will see, there is an enormous amount of information about the world, if just a little imagination and thinking are applied.

(Feynman et al. 1963, vol. 1, ch. 1-2)

The atoms are things, notably because they are discrete and thus countable (even if there were an infinite number of them) and because they are permanent - they do not come into existence and they do not go out of existence.

In virtue of the atoms being permanent, they can furthermore be considered as things in the sense of substances. Over and above that, for them to be substances, one can follow Aristotle in demanding that they exist independently of each other (cf. Categories, ch. 5). That is to say, no atom is ontologically dependent on other atoms - the atoms may be ontologically dependent on an absolute space, as in Democritean and Newtonian, or on God, as in Christian metaphysics; but if they claim an independent existence, that means that no atom is dependent for its existence on there being other atoms. If they exist independently of each other, each atom has some intrinsic features that characterize it as the atom that it is - that is, features that belong to each atom independently of whether or not there are other atoms (cf. the definition of intrinsic properties in Langton and Lewis 1998; see Hoffmann-Kolss 2010, part 1, for an extensive discussion of intrinsic properties). According to Aristotelian metaphysics, these intrinsic features are a form (eidos) that makes each thing the thing that it is (see Metaphysics, 
book 7) - at least as far as its being a thing of a certain kind is concerned, if not its being a certain individual (see Frede and Patzig 1988 for the latter view).

However, Aristotle does not have atoms in mind. His first and foremost candidate for substances are living organisms. Aristotelian forms then are intrinsic kind properties in the first place, namely those intrinsic properties that make it that a thing is a thing of a certain kind - such as tigers, lions or elephants in the case of living beings, or electrons, protons, neutrons in the case of candidate objects for atoms in the literal sense of indivisible objects in classical physics. In the latter case, properties such as a certain value of mass and charge (and spin in the quantum case) are the candidates for kind making properties. In any case, one does not find in physics candidates for intrinsic properties that go beyond kind making properties. For instance, all electrons have the same mass and charge. In other words, there are no intrinsic properties that distinguish and hence individuate the things that belong to the same natural kind.

If one searches for a property that individuates, the first and foremost candidate is position. That is to say, its position relative to other things individuates each thing in a configuration of things at any given time, since no two things can be at the same place at the same time. Moreover, the history of position - that is, the trajectory of each thing - provides for its identity in time, since no two things can have the same trajectory. However, position is not an intrinsic property. It is a relation, namely either the occupation relation between things and points or regions of space on absolutism about space, or the distance relation between things on relationalism about space. If one enquires into an intrinsic feature that individuates things, there is no qualitative property available. Nonetheless, one can go for a primitive thisness (haecceity) of each thing, namely the feature of being that thing conceived as primitive. But one has to pay a high metaphysical price for haecceitism: on this view, possible worlds are distinct simply by permutation of haecceities, without there being any qualitative difference between them (see Adams 1979).

Position is not only the first and foremost candidate for the feature that individuates things. It also is the first and foremost candidate for the world making relation. If there is a plurality of things, there has to be something that relates these things so that they make up a world. In other words, if one considers a plurality of worlds, there has to be something that relates the things in each world, by contrast to a relation that establishes a comparison between things across different possible worlds (such as e.g. the counterpart relation). At least as far as the actual world is concerned, position in the sense of spatial relation (distance) is what unites the world (cf. Lewis 1986, ch. 1.6). Ontologies that admit a plurality of things, but no relations (see Heil 2012, ch. 7, Lowe 2016) face the problem that there then is no relation that binds the many things together so that they make up a world. On this basis, one can make a case for putting relations before intrinsic properties: a relation of a certain type is indispensable as world making relation if one admits a plurality of things, and only a relation can individuate these things (if one dismisses haecceities). It turns out that position in the sense of the distance relation can fulfill both these tasks.

Moreover, taking physics into account, it is by no means obvious that the kind making properties are indeed intrinsic properties. Although parameters like mass and charge (as well as spin in quantum physics) are attributed to microphysical objects taking individually, this fact does not warrant the conclusion to considering them as intrinsic properties of the physical objects. The reason is that in physics, these parameters are exclusively considered in terms of 
their dynamical role in the laws of a given theory, namely in terms of what they do for the motion of the physical objects. Thus, commenting on Newton's Principia, Mach says that "the true definition of mass can be deduced only from the dynamical relations of bodies" (1919, p. 241). The same goes for charge, spin, etc. On this basis, one can make a case for considering these parameters to be relations rather than intrinsic properties, namely as expressing dynamical relations as encoded in the laws of a given physical theory (see Esfeld and Deckert 2017, ch. 2).

This case is strengthened when one turns to quantum physics: in brief, in quantum physics, also the classical parameters such as mass and charge are situated on the level of the wave function, which is the central dynamical parameter in quantum physics. The wave function cannot be considered as being or encoding intrinsic properties of physical objects due to its entanglement. That is to say, in brief, there is a wave function only for a configuration of objects - in the last resort, the configuration of the objects of the entire universe. Entanglement cannot be reduced to (or be replaced with) additional local variables of the physical objects that could be candidates for intrinsic properties, as was proven by Bell's theorem (1964, reprinted in Bell 1987, ch. 2). And if one admits additional variables in order to solve the famous measurement problem of quantum physics, the first and foremost candidate for such variables is position, which is, again, a relation. Thus, basing oneself on physics, one can make a case for relations instead of intrinsic properties.

The metaphysical stance that makes this case is known as ontic structural realism, with structures being conceived as concrete physical relations among physical objects that do not have any intrinsic properties. This does not imply that one either has to resort to haecceities as that what individuates objects or to come to the conclusion that there is nothing that individuates physical objects (so that there are no objects at all). Dynamical relations such as entanglement in quantum physics or mass and charge in classical physics cannot individuate objects - in the formalisms of physical theories, they presuppose (individual) objects as that to which they are applied and whose evolution they constrain. But position can individuate objects - as mentioned above, the objects in a configuration can be conceived as being individuated by the distance relations in which they stand. Thus, on the stance known as moderate ontic structural realism, there still are objects, but all there is to these objects are the relations in which they stand (see Esfeld and Lam 2011).

By contrast, on the stance known as radical ontic structural realism, the objects are dissolved in the structures in the sense that any putative object is supposed to turn out to be constituted by relations (see French and Ladyman 2003). The main objection to this stance is that structures are commonly defined over a set of objects. That is to say, there cannot be relations without relata that these relations relate. Even if this objection is sound, note that it is no argument for intrinsic properties, for it does not say anything against the relata being individuated by the relations in which they stand. In any case, radical ontic structural realism is not a thing ontology: it poses structures in the guise of relations instead of things. Moderate ontic structural realism, on the contrary, is a thing ontology, with its specific feature being the view that the things are individuated by the relations in which they stand and do not have intrinsic properties. Hence, in any case, ontic structural realism is opposed to Aristotelian metaphysics with its stress on intrinsic properties; in particular, it is diametrically opposed to the above mentioned view that there are no relations. Quite to the contrary, relations are all 
there is or at least all there is when it comes to the fundamental features of things and to what individuates things.

If relations individuate things, the things are no longer substances in the sense of independent things, since they are no longer ontologically independent of each other. If position in the sense of distance is the relation that individuates, any object is ontologically dependent on there being at least two further objects for it to be individuated by the distance relations that it bears to the other objects with which it makes up a world. The failure of ontological independence of the things in the world is the reason why Schaffer (2010) maintains ontological monism in the sense of the ontological priority of the whole: only the whole universe is a substance according to this criterion. But note that this is only a verbal issue (i.e. a matter of definition): by adopting the criterion of ontological independence for something to be a substance, we automatically get to the stance of ontological monism in the sense of the view that there is only one substance as soon as we take relations to be essential to the objects in the world (e.g. because they are individuated by relations). However, by thus getting to monism, we have not moved away from an ontology of a plurality of things. We only refuse to grant the things the status of substances. This monism light so to speak is to be contrasted with a substantial monism so to speak according to which there is only one object. In other words, what is at issue here, in the context of (moderate) ontic structural realism, is still atomism with a plurality of individual things, albeit considered as ontological monism of only one substance, in contrast to the non-thing ontology of there being only one continuous stuff.

\section{Discrete objects: events instead of substances}

Another change to the traditional thing ontology inspired by contemporary physics consists in representing the configuration of matter of the universe as being inserted not in threedimensional space, but in four-dimensional space-time. In this case, the configuration does not change in time; it is wholly present in space-time. In other words, everything that there is in space-time simply exists. Existence is not tied to a mode of time, such as only the present, or the present and the past existing and the future not existing, coming into existence (becoming) as time passes. The shift from space to space-time hence rules out in the first place ontologies that tie existence to a specific mode of time, such as presentism (see e.g. Saunders 2002); but it thereby also has implications for thing ontologies.

If things are inserted in space-time instead of in space, each material object, including its entire evolution, is conceived as occupying a certain region in space-time, namely what is known as a worldline (instead of a point or a region in space, with the occupied points or regions changing as time passes). This view implies the following shift in metaphysics: if things are inserted in three-dimensional space and change in time, they persist by enduring. They are wholly present at each point of time. Consequently, they do not have temporal parts; they only have spatial parts (and thus occupy regions of space), unless they are atoms in the sense of things that are located at points of space. By contrast, if things are inserted in fourdimensional space-time, they persist by perduring: they occupy a region in space-time, namely a worldline. Consequently, they do not only have spatial parts (unless they are atoms), but also temporal parts. The parts of the worldline of a thing are its temporal parts, and the existence of the thing as a whole is its entire worldline. 
More precisely, the shift in ontology then is the following one: one can consider discrete, enduring things as substances, now taken in the sense of objects that are wholly present at each moment of their existence. By contrast, if one takes things to be worldlines, one considers them to be processes in the sense of continuous sequences of events, because they have temporal parts. Events and processes are four-dimensional entities, whereas substances in the sense of endurants are three-dimensional entities. An ontology of such fourdimensional entities still is a thing ontology; it still is an ontology of discrete objects, but these are sequences of events (processes) instead of enduring substances (as regards fourdimensionalism, see e.g. Heller 1990, Sider 2001 and Benovsky 2006, first part).

The physics that suggests this shift in ontology is the special and the general theory of relativity. The reason for that shift is that in the special theory of relativity, in contrast to Newtonian physics and consequent upon the velocity of light being independent of a reference frame and thus absolute, there no longer is a unique temporal order of all the events in the universe. Spatial and temporal distances between events are relative to a reference frame, and there is no universally privileged reference frame. Only the four-dimensional, spatio-temporal interval between any two events is independent of the choice of a reference frame. This is the reason for considering all the events in the universe as being inserted in a four-dimensional space-time, resulting in what is known as the block universe metaphysics. According to this metaphysics, the universe is a single four-dimensional block with everything that there is in space and time simply existing in that block, that is, being located in that block by occupying a certain region in that block, namely a worldline. The absence of a privileged reference frame then means that there is no unique, privileged way of slicing the four-dimensional space-time block into three-dimensional, spatial hypersurfaces that are ordered in one-dimensional time (see e.g. Balashov 2010 for the case for the block universe metaphysics based on relativity physics).

The block universe metaphysics is not committed to the existence of a four-dimensional, absolute space-time into which the material things are inserted. It can also be conceived as a relationalism about space-time, namely in terms of four-dimensional, spatio-temporal relations between events and their continuous sequences, that is, the worldlines. The main challenge that the block universe metaphysics faces is to establish a distinction between variation within a given configuration of things and change of that configuration. In brief, the differences in the four-dimensional, spatio-temporal relations between events and continuous sequences of events (worldlines) provide for variation within the block universe, but they do not change, since they all exist at once; a fortiori, there is no becoming in the block universe (Geach 1965, in particular p. 323, is the main source for this objection).

The block universe metaphysics is inspired by the physical theories of relativity, but it is not imposed upon us by the physics. In the first place, one can be a scientific realist about that physics and still adopt the attitude that is also available for the Leibnizian relationalist when it comes to Newtonian mechanics, which is formulated in terms of an absolute space and time: the relationalist can regard the absolute space and time and all the absolute quantities that appear in Newtonian mechanics as the means to represent the (Leibnizian) distance relations among point particles throughout the history of the universe, instead of subscribing to an ontological commitment to them (see Huggett 2006). By the same token, the relationalist can regard the Minkowskian geometry of special relativity and the Riemannian geometry of general relativity as the means to represent the basic ontology of (Leibnizian) distance 
relations among elementary discrete objects and the change in these relations throughout the history of the universe (see Esfeld and Deckert 2017, chs. 5.2-4). Furthermore, both in the classical, Newtonian case as well as in the relativistic case, there is an alternative formulation of the physics available that in the end yields the same empirical results based on an ontology of Leibnizian distance relations, namely Barbour's shape dynamics (see e.g. Barbour and Bertotti 1982, Barbour 2012 and Gryb and Thébault 2016).

Be that as it may, as regards an ontology of discrete objects in terms of a thing ontology, conceiving the things as being inserted into a three-dimensional space with change in onedimensional time or conceiving them as being inserted into a four-dimensional block of space-time is no big issue. The things still are substances in the sense of being permanent and the considerations about their ontological dependence or independence apply in the same manner as mentioned in the preceding section. In the case of four-dimensionalism, the things are not substances only in the sense that they are perdurants instead of endurants.

The big issue, if there is a big issue here, is the possibility to maintain an ontology of discrete objects, but to abandon the assumption that the objects persist. If they do not persist, they no longer are things - and a fortiori not substances -, but there still is a plurality of discrete objects that are individuated by their spatio-temporal location. These then are single events. On this ontology, there are no continuous sequences of events and hence no worldlines and no processes. There just are discrete, single events. If there are enough of such events and if they are close enough to each other, it may be possible to explain our experience of macroscopic objects that persist.

These events are ephemeral. If one formulates this ontology in terms of threedimensionalism, these events come into being out of nothing and they instantaneously are annihilated - there is no change in anything, since there is nothing that persists. If one formulates this ontology in terms of four-dimensionalism (the block universe metaphysics), then some points of space-time are occupied by material events, and these events are distributed in a discrete manner in continuous space-time - there is empty space-time between any two material events, thus no continuous sequences of events.

Such an ontology is not only a possible position in the logical space of philosophical ontologies. It can also be based on physics, in this case a formulation of quantum physics with a dynamics of wave function collapse. In brief, on this view, whenever the quantum wave function collapses in the mathematical space on which it is defined, there occurs a single event at a point in physical space; these events are known as flashes (see Tumulka 2006, p. 826, and see Bell 1987, ch. 22, for the original proposal, as well as Allori et al. 2008, section 3.2 , and 2014, section 2.3). The claim then is that these discrete flashes are all there is in physical space. As any ontology based on quantum physics, this ontology is in dispute (see Arntzenius 2012, ch. 3.15, for an endorsement and Maudlin 2011, ch. 10, and Esfeld and Deckert 2017, ch. 3.3, for a critical discussion). The interest for metaphysics is that this ontology illustrates the possibility of subscribing to a genuine event ontology - that is, an event ontology that cuts philosophical ice by admitting only single, discrete events that hence are discrete objects, but not substances because they do not persist (by contrast to a reformulation of a substance ontology in terms of continuous sequences of events, as in the case of the block universe metaphysics based on relativity physics). 


\section{Continuous stuff}

The alternative to an ontology of a plurality of discrete objects is an ontology of one continuous object. There hence is only one substance that is permanent and that, since it is the only object in the universe, is not ontologically dependent on any other object. This ontology can also be traced back to the Presocratics, more precisely to the first Presocratic natural philosophers such as Thales, Anaximander and Anaximenes, who can all be interpreted as searching for the one stuff out of which the universe is made. On this ontology, there is, in contrast to a plurality of discrete, finally indivisible objects (atoms), just one continuous stuff that fills all of space. This stuff is known as gunk. However, like the ontology of discrete objects, this ontology is not committed to subscribing to an absolute background space: there can just be one extended, continuous stuff, but there has not have to be a space or space-time that is distinct from that stuff and into which it is inserted. This stuff can be conceived as being infinitely divisible. By contrast to the ontology of atoms, which are indivisible and therefore point particles, the stuff ontology does not have commit itself to points, neither point-objects (atoms), nor points of space (see Arntzenius and Hawthorne 2005).

If there is just stuff, the only variable is the density of that stuff. That is to say, in order to account for variation, one has to admit different degrees of density of stuff at different points or in different regions of space, with these degrees of density changing in time. The primitive stuff is a bare substratum that, moreover, admits of different degrees of density in different points or regions of space as a primitive matter of fact: there is nothing that accounts for the difference between the degrees of density of the stuff in different regions of space (mass, for instance, would, again, be a dynamical variable, not an intrinsic property of the stuff - see Allori et al. 2014, pp. 331-332). Apart from the commitment to a bare substratum of matter, the main challenge for this ontology is to formulate a dynamics for the evolution of the density of stuff such that stuff is concentrated in certains regions of space in a manner that the experience of discrete objects is accounted for. In other words, our experience of discrete objects down to molecules and atoms in the form of the chemical elements does not impose an ontology of a plurality of discrete objects on us. There may be just one object in the guise of a continuous stuff on the fundamental level. But any such ontology then has to achieve the formulation of a dynamics for that stuff that allows it to be distributed in a way as if there were not only discrete macroscopic, but also discrete microscopic objects down to the chemical elements, if not the quantum particles.

Whereas the ontology of discrete objects is supported by particle physics, the ontology of a continuous stuff can draw on fields in physics. However, fields enter into modern physics as mediators of particle interactions, like the electromagnetic field in the Maxwell-Lorentz theory of the electrodynamic interaction in the $19^{\text {th }}$ century. Nonetheless, one can claim that in the subsequent development of physics, fields have become ever more important and gained an autonomous status, such as the metrical field in general relativity theory, which is also the gravitational field. That notwithstanding, physics has not replaced particles with fields: the fields in quantum physics (quantum field theory) are not fields that have values at the points of space (or space-time), and quantum field theory is employed to formulate today's standard model of particle physics (one can even go as far as maintaining an ontology of permanent particles for quantum field theory, see Esfeld and Deckert 2017, ch. 4).

Nevertheless, there is a formulation of quantum physics available that works with a dynamics of the collapse of the wave function and that regards the quantum mechanical wave 
function as referring to a matter density field in physical space and as describing the evolution of this field - which is then such that, upon collapse of the wave function, the matter density concentrates itself at certain points or regions of space so that precise measurement outcomes and, in general, the experience of discrete objects is accounted for (see Ghirardi et al. 1995 and Allori et al. 2008, section 3.1, and 2014, section 2.2). However, it is in dispute whether this proposal really succeeds in accommodating precise measurement outcomes. Moreover, in order to account for correlated measurement outcomes across space, this proposal has to postulate that the matter density can be instantaneously delocated across arbitrary distances in physical space (corresponding to the instantaneous collapse of the wave function all over space) (see Egg and Esfeld 2015). These reservations notwithstanding, this worked out proposal for an ontology of quantum physics in terms of a matter density field shows that a non-thing ontology in the guise of an ontology of one continuous stuff is a live option in contemporary metaphysics.

Such an ontology does not necessarily have to be conceived of in terms of only one continuous stuff that is extended all over space with different degrees of density. Rovelli (1997) proposes in the context of general relativistic physics an ontology of a plurality of stuff fields that overlap and interact with each other, such as the gravitational field and the electromagnetic field both conceived as primitive stuff fields that stretch all over space. In this case, the difference between these fields consists in differences in their dynamics, that is, in differences in their characteristic evolutions of concentrating and stretching out.

\section{Conclusion}

There is evidence of a plurality of discrete objects, in common sense as well as in science, where the theory of the atomic constitution of matter is the basis of the success of modern science from physics to molecular biology. If one takes this evidence to be a good argument for endorsing an ontology of a plurality of discrete objects, the formulation of that ontology then depends on the view that one adopts with respect to space and time: a plurality of enduring substances, or a plurality of worldlines (processes) that are continuous sequences of events, being perduring objects. In both cases, one defends an ontology of a plurality of things. One gets to a non-thing ontology if one admits instead of continuous sequences of events only a plurality of single, discrete events. These still are discrete objects, but they are ephemeral (flashes). The main version of a non-thing ontology, however, is the ontology of a continuous stuff that stretches all over space. Both this non-thing ontology and the ontology of a plurality of discrete objects can be traced back to the first Presocratic philosophers. They remain live options for an ontology that takes today's physics into account.

\section{References}

Adams, Robert M. (1979): "Primitive thisness and primitive identity”. Journal of Philosophy 76, pp. 5-26.

Allori, Valia, Goldstein, Sheldon, Tumulka, Roderich and Zanghì, Nino (2008): "On the common structure of Bohmian mechanics and the Ghirardi-Rimini-Weber theory". British Journal for the Philosophy of Science 59, pp. 353-389.

Allori, Valia, Goldstein, Sheldon, Tumulka, Roderich and Zanghì, Nino (2014): "Predictions and primitive ontology in quantum foundations: a study of examples". British Journal for the Philosophy of Science 65, pp. 323-352.

Arntzenius, Frank (2012): Space, time and stuff. Oxford: Oxford University Press.

Arntzenius, Frank and Hawthorne, John (2005): “Gunk and continuous variation”. The Monist 88, pp. 441-465. 
Balashov, Yuri (2010): Persistence and spacetime. Oxford : Oxford University Press

Barbour, Julian B. (2012): "Shape dynamics. An introduction”. In: F. Finster, O. Müller, M. Nardmann, J. Tolksdorf and E. Zeidler (eds.): Quantum field theory and gravity. Basel: Birkhäuser. Pp. 257-297.

Barbour, Julian B. and Bertotti, Bruno (1982): "Mach's principle and the structure of dynamical theories". Proceedings of the Royal Society A 382, pp. 295-306.

Bell, John S. (1987): Speakable and unspeakable in quantum mechanics. Cambridge: Cambridge University Press.

Benovsky, Jiri (2006): Persistence through time, and across possible worlds. Frankfurt (Main): Ontos.

Egg, Matthias and Esfeld, Michael (2015): "Primitive ontology and quantum state in the GRW matter density theory". Synthese 192, pp. 3229-3245.

Esfeld, Michael and Deckert, Dirk-André (2017): A minimalist ontology of the natural world. New York: Routledge.

Esfeld, Michael and Lam, Vincent (2011): "Ontic structural realism as a metaphysics of objects". In: A. and P. Bokulich (eds.): Scientific structuralism. Dordrecht: Springer. Pp. 143-159.

Feynman, Richard P., Leighton, Robert B. and Sands, Matthew (1963): The Feynman lectures on physics. Volume 1. Reading (Massachusetts): Addison-Wesley.

Frede, Michael and Patzig, Günther (1988): Aristoteles 'Metaphysik Z'. Band 2: Kommentar. München: Beck.

French, Steven and Ladyman, James (2003): "Remodelling structural realism: quantum physics and the metaphysics of structure". Synthese 136, pp. 31-56.

Geach, Peter (1965): “Some problems about time”. Proceedings of the British Academy 51, pp. 321-336.

Ghirardi, Gian Carlo, Grassi, Renata and Benatti, Fabio (1995): "Describing the macroscopic world: closing the circle within the dynamical reduction program". Foundations of Physics 25, pp. 5-38.

Graham, Daniel W. (2010): The texts of early Greek philosophy. The complete fragments and selected testimonies of the major Presocratics. Edited and translated by Daniel W. Graham. Cambridge: Cambridge University Press.

Gryb, Sean and Thébault, Karim P. Y. (2016): “Time remains”. British Journal for the Philosophy of Science 67, pp. 663-705.

Heil, John (2012): The universe as we find it. Oxford: Oxford University Press.

Heller, Mark (1990): The ontology of physical objects: four-dimensional hunks of matter. Cambridge: Cambridge University Press.

Hoffmann-Kolss, Vera (2010): The metaphysics of extrinsic properties. Frankfurt (Main): Ontos.

Huggett, Nick (2006): “The regularity account of relational spacetime”. Mind 115, pp. 41-73.

Langton, Rae and Lewis, David (1998): "Defining 'intrinsic”". Philosophy and Phenomenological Research 58, pp. 333-345.

Lewis, David (1986): On the plurality of worlds. Oxford: Blackwell.

Lowe, E. Jonathan (2016): “The are (probably) no relations". In: A. Marmodoro and D. Yates (eds): The metaphysics of relations. Oxford: Oxford University Press. Pp. 100-112.

Mach, Ernst (1919): The science of mechanics: a critical and historical account of its development. Fourth edition. Translation by Thomas J. McCormack. Chicago: Open Court.

Maudlin, Tim (2011): Quantum non-locality and relativity. Third edition. Chichester: Wiley-Blackwell.

Rovelli, Carlo (1997): "Halfway through the woods: contemporary research on space and time". In: J. Earman and J. Norton (eds.): The cosmos of science. Pittsburgh: University of Pittsburgh Press. Pp. 180-223.

Saunders, Simon (2002): "How relativity contradicts presentism”. In: C. Callender (Hg.): Time, reality and experience. Cambridge: Cambridge University Press. Pp. 277-292.

Schaffer, Jonathan (2010): "Monism: the priority of the whole”. Philosophical Review 119, pp. 31-76.

Sider, Theodore R. (2001): Four-dimensionalism. An ontology of persistence and time. Oxford: Oxford University Press.

Tumulka, Roderich (2006): “A relativistic version of the Ghirardi-Rimini-Weber model”. Journal of Statistical Physics 125, pp. 825-844. 